\title{
Young-age prostate cancer
}

\author{
S Hussein, ${ }^{1}$ S Satturwar, ${ }^{2}$ T Van der Kwast ${ }^{2}$
}

${ }^{1}$ Department of Laboratory Medicine, Kuwait Cancer Control Center, Kuwait, Kuwait ${ }^{2}$ Department of Pathology, Princess Margaret Cancer Centre, University Health Network, Toronto, Ontario, Canada

\section{Correspondence to}

Professor Theo $\mathrm{H}$ Van der Kwast, Department of Pathology, Princess Margaret Cancer Centre, University Health Network, Toronto, Ontario M5G 2M9, Canada; theodorus.vanderKwast@uhn.ca

Received 3 March 2015 Accepted 5 March 2015 Published Online First 2 April 2015
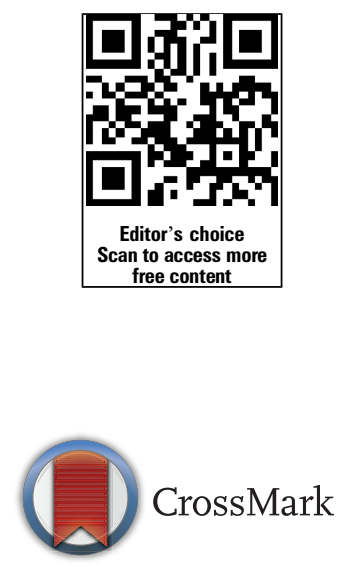

To cite: Hussein $S_{\text {, }}$ Satturwar S, Van der Kwast T. J Clin Pathol 2015;68:511-515.

\section{ABSTRACT}

Although prostate cancer is a disease of the elderly, its diagnosis is not uncommonly made in men younger than 55 years. Both ethnic, familial and genetic factors play a role in the early onset of prostate cancer, but the biology, particularly of low-grade prostate cancers detected at young age is not well understood. Autopsy studies have shown a high prevalence of Gleason score 6 prostate cancers in men under 55 years, but mortality of prostate cancer at this young age is almost negligible. Recently, a number of susceptibility genes such as BRCA2 and HOXB13 were reported, each with their own specific biological and histopathology features. In this review, we provide an update on the most recent findings in young-age prostate cancer.

\section{INTRODUCTION \\ Overview}

Prostate cancer is a disease of the elderly with most men diagnosed at the age of 65 years or above. ${ }^{1}$ The incidence of prostate cancer rapidly increases after the age of 55 years. ${ }^{1-3}$ During the past few decades, the median age of diagnosis was steadily decreasing, as a consequence of the widespread introduction of prostate-specific antigen (PSA) screening. In the USA (Surveillance Epidemiology and End Result (SEER) data), the median age at diagnosis of prostate cancer dropped from 72 years in 1986 to 66 years in $2011 .^{2}$ Since 1977, there was an overall threefold increase of prostate cancer incidence; however, among men aged $<55$ years in the UK this increase was ninefold. ${ }^{1}$ Likely, this trend change is because more men at young age underwent PSA testing, particularly when they would have a positive family history. Furthermore, a baseline PSA testing at the age of 40 years was recommended by American Urological Association, ${ }^{4}$ in 2009 , as some studies had indicated that increased PSA value at this age would herald the development of a more aggressive cancer at older age. ${ }^{5}$ The substantial increase of prostate cancer diagnosis at young age raises today a number of important questions both about their biology and their management. A 60-year-old man with a low-risk, biopsy Gleason score 6, prostate cancer is a suitable candidate for active surveillance, who could defer therapy until signs of grade progression or rapid growth. However, a similar scenario in a 50-year-old man might prompt immediate intervention, taking into account his longer life expectancy and the suspicion that prostate cancer detected at young age might behave more aggressively. ${ }^{7-10}$ This review intends to identify characteristic features of prostate cancer in men at young age as opposed to prostate cancers identified in older men, taking into consideration both histomorphological and molecular-genetic findings.

\section{Prevalence}

Autopsy studies can give an insight into the prevalence of asymptomatic or latent prostate cancer in a population. The prevalence of latent prostate cancer in younger men varies markedly among different autopsy series. Breslow et al ${ }^{11}$ showed that, on average, about $15 \%$ of men aged $45-54$ years have latent prostate cancer with a prevalence ranging from $9 \%$ to $22 \%$, depending on the geographical distribution of the population. Sakr et $\mathrm{al}^{12}$ reported that $27 \%$ and $34 \%$ of men in USA aged $30-40$ years and $40-50$ years, respectively, harboured a prostate cancer, although there is none with a high-grade component. In a Greek autopsy series, prostate cancer was found only in one among 38 men aged $40-50$ years $(2.6 \%) .{ }^{13}$ However, in another Mediterranean (Spanish) population, a prevalence of $14.3 \%$ was established in this age group. ${ }^{14}$ These observations are in contrast to the much higher prevalence of $27 \%$ and $34 \%$ found in men aged 40-50 years from Hungary and the USA, respectively. ${ }^{12}{ }^{15}$ Altogether, these observations would indicate that about 20\%-30\% of older men aged $40-50$ years living in the USA and Middle and Northern Europe would harbour a prostate cancer. Importantly, their natural course and biology remain unclear.

\section{Definition of young-age and early-onset prostate cancer}

The age cut-off to define young-age prostate cancer is somewhat arbitrary. The upper limit of the age range for young-age prostate cancer cited in the literature varies between 50 and 55 years. According to UK and USA epidemiological data, the mortality from prostate cancer at the age under 55 years is extremely low, but starts rising rapidly in men over 55 years. On the other hand, autopsy studies show a high prevalence of latent prostate cancer in the fourth and fifth decades, ${ }^{12}$ some of which could be detectable in case of random prostate biopsies prompted by elevated PSA. The European and North American urology associations recommended PSA testing particularly for men aged over 55 years, and as a consequence, it is likely that in the PSA era a larger proportion of prostate cancers discovered in European and American men aged under 55 years were symptomatic as compared with men aged over 55 years. Obviously, a prostate cancer discovered because of the symptoms of metastatic disease is an entirely different disease than a prostate cancer identified merely on the basis of a PSA prompted prostate needle biopsy. The literature occasionally refers to early-onset prostate cancer as a subset of young-age or familial prostate cancers with more aggressive disease and higher prostate-cancer-specific death. ${ }^{7-10}$ Here, we would propose to define early-onset prostate 
cancer as prostate cancers detected in men aged $<55$ years with at least one clinical sign of prostate cancer, such as positive digital rectal examination or a visible tumour at time of imaging (at least T2 prostate cancer) rather than a prostate cancer, incidentally detected because of PSA testing alone (T1c). In contrast, young-age prostate cancer could be defined broadly as any prostate cancer, regardless tumour extent or clinical manifestations in men aged $<55$ years. Although this distinction between young-age and early-onset prostate cancer is not always explicit in the literature, we tried to apply these two definitions for this review on the basis of the populations studied or data provided in the reviewed publications.

\section{CLINICAL FEATURES \\ Aggressiveness}

Age at diagnosis of cancer is a well-recognised prognostic factor in a patient diagnosed with malignancy. Review of the literature indicates that earlier detection of the prostate cancer with lowgrade and stage disease in young men has a superior disease outcome. The better tolerance for aggressive therapy and fewer comorbid conditions account for a better outcome with superior biochemical recurrence rates for younger men compared with older men. ${ }^{16-18}$ Although few studies have demonstrated an association of very young age and high-stage disease, it is unclear whether early age at diagnosis adversely influences the outcome in a young patient with prostate cancer. ${ }^{7-10}$ Huben et al analysed in the pre-PSA era data on 20156 prostate cancer cases from American College of Surgeon's National Survey and showed significantly better disease-specific survival for younger patients with early-onset localised disease at 5 and 10 years of follow-up. Survival was slightly better in younger patients at 5 years for other stages of disease. Although prostate cancer is more likely to be metastatic at the time of diagnosis in men aged $<50$ years, there did not appear to be any significant biological differences in prostate cancer in men aged $<50$ years as compared with older men. ${ }^{7}$ A more recent analysis of 453195 prostate cancer cases by using the SEER data identified 30338 (9\%) prostate cancer cases in men aged 35-54 years. The younger men were more commonly African-American, were less likely to have a high-grade tumour, but paradoxically also less likely to be diagnosed with organ-confined disease. Disease-specific survival was similar for older and younger men with a Gleason score 5-7 prostate cancer. However, among the men with high-grade locally advanced disease, the younger men were three times more likely to die of prostate cancer than older men. In the subset of men with pathologically confirmed highgrade and advanced stage disease who had undergone a prostatectomy, the younger men were at least five times more likely to die of prostate cancer than another age group. ${ }^{9}$

\section{PSA-tested populations}

The European Randomized Study of Screening for Prostate Cancer (ERSPC) is a population-based screening trial which reported about $20 \%$ reduction in prostate cancer mortality at 11 and 13 years of follow-up. The result was based on a $3 \mathrm{ng} / \mathrm{mL}$ PSA as a cut-off value for biopsy indication. ${ }^{19}$ Although the target age group for the ERSPC was men aged between 55 and 69 years, the Swedish component of the trial had a lower limit of 50 years. Among the age group of 50-55 years in this screening trial centre, only six cancers $(2 \%)$ were found, all of them with a Gleason score 6 prostate cancer during the prevalence screening round. ${ }^{20}$ Data from the Department of Defense registry (USA) study showed that $96 \%$ of prostate cancers detected in mandatory PSA screened men aged under 55 years were
Gleason score 6 prostate cancers. The remainder 4\% showed a Gleason score $7 .^{21}$ These findings are in clear contrast with those of an Australian study in an opportunistic PSA testing setting: at a cut-off PSA value of $4 \mathrm{ng} / \mathrm{mL}, 44 \%$ of the identified prostate cancers in the age group $<55$ years were Gleason score 6 prostate cancers, while $31 \%$ and $7 \%$ were Gleason score 7 and Gleason score $>7$ prostate carcinomas, respectively. ${ }^{22}$ An essential difference in the Australian PSA tested population with the ERSPC and Department of Defense (USA) populations is that the former represented opportunistic, instead of a population-based or mandatory PSA testing. As a consequence, men in the Australian population with a family history of prostate cancer and/or more awareness of prostate cancer were more likely to get PSA tested, which may explain its increased proportion of more aggressive prostate cancer.

\section{RISK FACTORS \\ Ethnicity}

Ethnic differences in prostate cancer incidence and outcome may result from the interaction of epidemiological and genetic factors. The highest reported incidence rate for prostate cancer is among African-American and Jamaican men of African descent. It is well recognised that black men are at a higher risk of being diagnosed with advanced stage prostate cancer at a young age. ${ }^{23-25}$ African-American men present their prostate cancer 2-3 years earlier than white Caucasian men. ${ }^{26} 27$ Powell et $a l^{23}$ reported more advanced disease and more frequent biochemical recurrence among young African-American compared with Caucasian men. Their recent (2014) analysis of SEER data demonstrated that African-American men at the age of 40-49 years had higher Gleason score and 2.8-3.2 times higher mortality and metastatic disease compared with American men of European descent of the same age group. These observations suggest a higher incidence of aggressive and rapidly growing early-onset prostate cancer in American-African men. The authors of this study speculated that the aggressiveness of prostate cancer in African-American men may be due to increased expression of genes associated with lethal prostate cancer like fatty acid synthase gene and higher expression of inflammatory cytokines, such as interleukin 6 (IL6), IL8 and IL 1B. ${ }^{25}$

\section{Familial prostate cancer}

Familial clustering of prostate carcinoma is the strongest determinant for prostate cancer risk. About 5\%-10\% of prostate cancers are hereditary and show a Mendelian pattern of inheritance just like in breast and colon cancer. ${ }^{28}$ The familial prostate cancer manifests at a younger age, usually 6-7 years earlier than sporadic disease and the risk of (familial) prostate cancer increases when a family member develops the disease at a younger age. ${ }^{29}$ If a father is diagnosed with prostate cancer prior to 60 years of age, the relative risk (RR) for developing prostate cancer increases from 1.5 to 2.5 . The RR increases to four times when there are two or more affected first-degree relatives, diagnosed at any age $(\mathrm{RR}=4.39 ; 95 \% \mathrm{CI} 2.61$ to 7.39)..$^{3031}$

\section{Susceptibility genes}

Genome wide association studies have revealed several single nucleotide polymorphisms in the germline DNA associated with an increased risk of prostate cancer, including a few associated with young-age prostate cancer. ${ }^{32-34}$ However, highly penetrant susceptibility genes causing young-age or early-onset prostate cancer have not yet been identified. Some of the rare genetic 
predisposition loci with small-to-moderate penetrance contributing to young-age prostate cancer are summarised in Table 1.

\section{$B R C A$ gene mutations}

Carriers of the BRCA 1 and 2 mutations are at risk for breast carcinomas and several other carcinomas. Although there is controversy about the increased risk of BRCA1 carriers for prostate cancer, all studies consistently reported that BRCA2 carriers have increased risk of more aggressive prostate cancer at younger age. ${ }^{35-41}$ The strongest predictors for the presence of a germline mutation of BRCA2 genes in patients with prostate cancer are a young age at onset and a family history of breast and/or ovarian cancer. Nevertheless, its contribution to youngage prostate cancer is limited. BRCA2 mutation is only found in $2 \%$ of prostate cancer cases diagnosed at the age of $\leq 55$ years. ${ }^{37}$ On the other hand, in their study of 263 men with a known $B R C A$ mutation, BRCA2 carriers had an estimated 23-fold increased risk of prostate cancer in men aged $<55$ years. Furthermore, Kote-Jarai et $a l^{38}$ reported an RR of 1.8 -fold to 4.5-fold for BRCA1 carriers and 2.5-fold to 8.6-fold increase for $B R C A 2$ carriers at the age of $\leq 65$ years. Similarly, the Icelandic founder mutation BRCA2 999 del5 is a specific mutation associated with prostate cancer at younger age and with poor survival. ${ }^{39}$ BRCA1/2 carriers have been shown to present with early-onset and aggressive disease, characterised by higher rates of lymph node involvement, distant metastasis at diagnosis and a higher mortality rate as compared with non-carriers. ${ }^{36} 40$ Histology of patient-derived xenografts from BRCA2 mutation carriers, high-risk breast cancer $B R C A$ negative families and sporadic prostate cancer cases revealed that a substantial proportion of them carried the features of intraductal carcinoma (figure 1) in association with conventional acinar adenocarcinoma. Thus, BRCA2 mutation carriers displayed the histopathological features of intraductal carcinoma in $14 / 33$ cases (42\%) as compared with patients with $B R C X(16 / 62,25.8 \%)$ and sporadic prostate cancer cases $(3 / 32,9 \%)$. Moreover, $64 \%$ of the BRCA2 carriers with intraductal carcinoma of the prostate had T3-T4 disease and poor outcome even after treatment with curative intent, in line with its more aggressive biology. ${ }^{41}$

Table 1 Features of most common germ-line aberrations of young-age prostate cancer

\begin{tabular}{|c|c|c|c|}
\hline & BRCA1 & $B R C A 2$ & НОХВ13 \\
\hline Genomic location & $17 q 21$ & $13 q 12.3$ & $17 q 21-22$ \\
\hline $\begin{array}{l}\text { Relative risk of } \\
\text { prostate cancer }\end{array}$ & $\begin{array}{l}1.8-4.5 \text { for age } \\
<65 \text { years }\end{array}$ & $\begin{array}{l}23 \text { for age }<55 \\
\text { years } \\
2.5-8.6 \text { for age } \\
<65 \text { years }\end{array}$ & $\begin{array}{l}\text { Eightfold for age } \\
<55 \text { years }\end{array}$ \\
\hline $\begin{array}{l}\text { Ethnic } \\
\text { predominance }\end{array}$ & $\begin{array}{l}\text { Ashkenazi } \\
\text { Jewish, } \\
\text { Norwegian, } \\
\text { Dutch and } \\
\text { Icelandic people }\end{array}$ & $\begin{array}{l}\text { Ashkenazi } \\
\text { Jewish, } \\
\text { Norwegian, } \\
\text { Dutch and } \\
\text { Icelandic people }\end{array}$ & Finnish, Swedish \\
\hline $\begin{array}{l}\text { Specific mutation } \\
\text { associated with } \\
\text { young-age } \\
\text { prostate cancer }\end{array}$ & - & $\begin{array}{l}\text { BRCA2 999del5 } \\
\text { (Icelandic } \\
\text { founder } \\
\text { mutation) }\end{array}$ & $\begin{array}{l}\text { HOXB13 G84E, } \\
\text { rs138213197 }\end{array}$ \\
\hline $\begin{array}{l}\text { Characteristic } \\
\text { histological } \\
\text { features }\end{array}$ & - & $\begin{array}{l}\text { Intraductal } \\
\text { carcinoma in } \\
42 \%\end{array}$ & $\begin{array}{l}\text { Pseudohyperplastic } \\
\text { features in } 45 \%\end{array}$ \\
\hline Prognosis & Unfavourable & Unfavourable & Favourable \\
\hline $\begin{array}{l}\text { Other associated } \\
\text { cancers }\end{array}$ & Breast and ovary & Breast and ovary & Breast \\
\hline
\end{tabular}

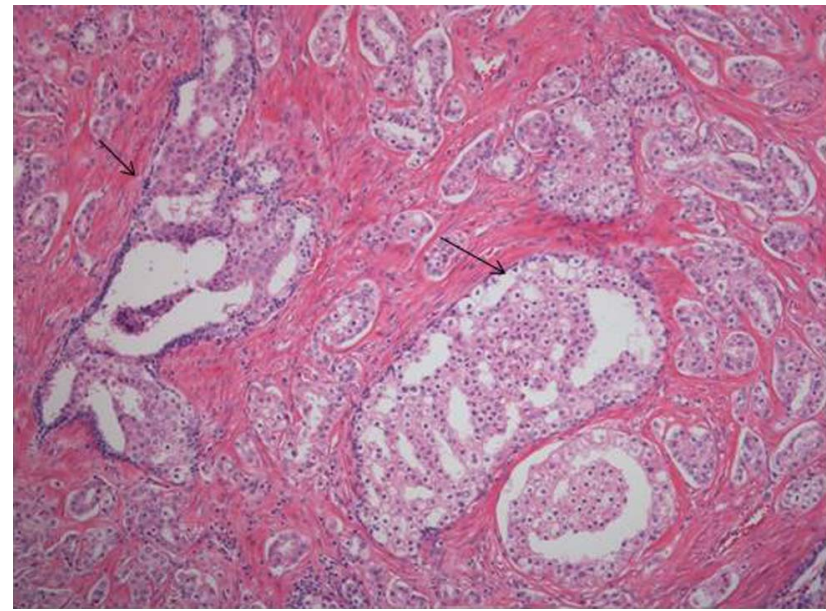

Figure 1 Histopathology of intraductal carcinoma (arrows) as can be identified in about $42 \%$ of BRCA2-associated prostate cancers.

\section{HOXB13 mutations}

Ewing $e t a l^{42}$ identified a rare but recurrent germline mutation c.252G->A (p.G84E, rs138213197), in the first exon of the homeobox B13 associated with increased risk of non-aggressive prostate cancer at young age and with positive family history. The mutation is primarily seen in West European people; however, different HOXB13 mutations have been detected in prostate cancer cases of African and Asian ancestry. A carrier frequency of G84E mutation among European-American with young-age familial prostate cancer was found to be $3.1 \%$, but its frequency was threefold higher (8.4\%) for both the Finnish and Swedish population. ${ }^{42} 43$ Although uncommon, this mutation accounts for an eightfold increased risk of prostate cancer diagnosis at the age of 55 years or less. This mutation was found to be more associated with hereditary prostate cancer (OR: 6.6; 95\% CI 3.3 to 12.0$).{ }^{44}$ HOXB13 interacts with androgen receptors (ARs) to promote development and differentiation of normal and cancerous prostate. It may act both as a tumour suppressor gene and as an oncogene, but the exact mechanism of HOXB13 in carcinogenesis remains unknown. ${ }^{42}$ In a Finnish population, HOXB13 G84E mutation was associated with young age ( $<55$ years), familial prostate cancer and elevated PSA of $20 \mathrm{ng} / \mathrm{mL}$ or more at the time of diagnosis. However, it was not associated with other clinical features related to disease aggressiveness, such as higher Gleason score, prostate cancer progression or recurrence. ${ }^{43}$ Review of the histomorphological features and molecular subtypes of prostatectomy specimens in 23 patients with prostate cancer with $\mathrm{HOXB13}$ G84E mutation carriers revealed a more frequent occurrence of (Gleason pattern 3) pseudohyperplastic-variant carcinoma (figure 2) and a markedly low prevalence of ERG + cancers (17\% of dominant tumour foci). The majority of the cases had Gleason score 7 prostate cancer (54\%), whereas 23\% were Gleason score 6 and $14 \%$ Gleason score $>8$. Their findings suggest that this could be a novel molecular pathway for carcinogenesis in HOXB13 carriers with more favourable outcome. ${ }^{45}$

\section{Pathway analysis}

The molecular basis of young-age prostate cancer is not yet understood. The integrative genomic analysis of 11 early-onset prostate cancer cases by Weischenfeldt et al revealed a key role of the androgen-androgen receptor axis as reflected by an increased frequency of the androgen regulated transmembrane 


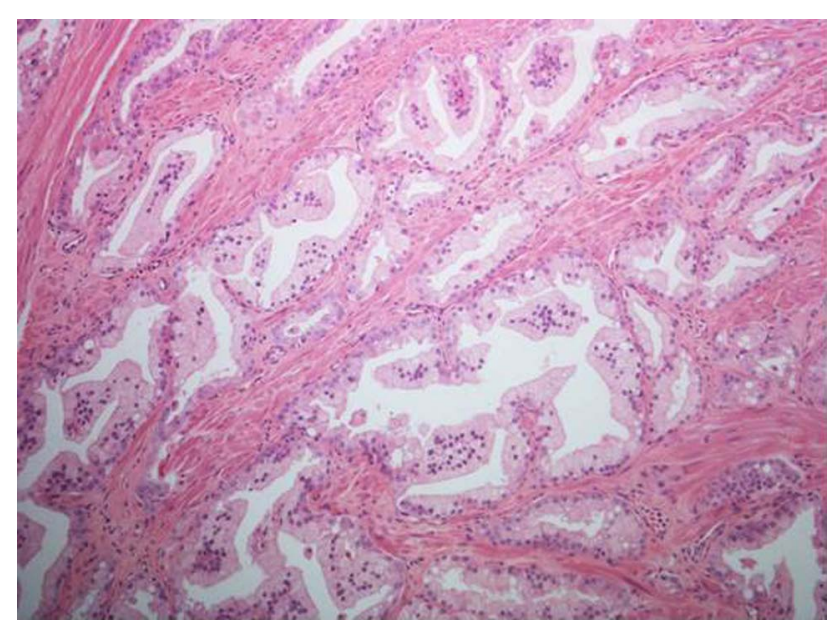

Figure 2 An example of pseudohyperplastic variant of prostate cancer, characteristic for HOXB13-associated prostate cancers.

serine protease 2 (TMPRSS2) gene fusion with the ERG gene in these cancers. This fusion is a very early event in prostate carcinogenesis and thought to be driven by increased androgen stimulation, ${ }^{46}$ The age dependency of ERG expression was further confirmed in this study using a tissue micro array of a large number of prostatectomy samples, including low-risk prostate cancers. In addition, the young-age prostate cancers demonstrated a significantly increased tumour AR level and positive correlation of AR levels with ERG rearrangements. ${ }^{46}$ The authors suggested a specific pathogenesis for young-age prostate cancers, distinct from the classical elderly-onset prostate cancers, although their proliferative kinetics as judged from the MIB-1 labelling index were the same as in elderly-onset prostate cancer. In a separate study, Schaefer et al analysed ERG protein overexpression in 1039 radical prostatectomy specimen in a PSA screened population and they similarly found a much higher frequency of $E R G+$ prostate cancer for the age group of $35-55$ years (ie, 64\%) as compared with $41 \%$ in the age group of 56-67 years. The ERG-positive status was significantly associated with young age at diagnosis, low-serum PSA and lower prostate volume but not with disease progression or biochemical tumour recurrence. ${ }^{47}$ Huang et al analysed ERG protein overexpression in a cohort of 121 men diagnosed with localised prostate cancer at $<50$ years of age and reported similar high frequency (64\%) of ERG-positive prostate cancers. In this study, the ERG-positive status was significantly associated with Gleason score and higher biochemical relapse rate but not with presurgical PSA levels, tumour volume, pathological stage, surgical margin or lymphovascular invasion. ${ }^{48}$ Steurer et $a l^{49}$ further confirmed the strong age dependency of TMPRSS2-ERG fusion for Gleason score $<7(4+3)$ cancers in young patients, while this age dependency was lost for higher Gleason score prostate cancers. In the same patient series, the age-dependent loss of the PTEN suppressor gene was only observed in ERG-positive prostate cancers, while the age-dependent loss of $5 \mathrm{q} 21$ and $6 \mathrm{q} 15$ was only noted in ERG-negative prostate cancers. The latter findings may be interpreted as evidence for a distinct genomic pathway of young-age TMPRSS2-ERG fusion positive and negative prostate cancers, the former pathway being the more dominant in low-grade young-age prostate cancers.

\section{CONCLUSIONS}

Autopsy studies have shown that in West-European and American men, the prevalence of prostate cancer at the age of
40-50 years is about $20 \%-30 \%$. Most, but not all studies show that PSA-detected prostate cancers at age under 55 years are low grade. More aggressive early-onset prostate cancer is seen more frequently in African-American men than in Caucasian men. Young-age prostate cancer is associated with a considerably increased risk of prostate cancer in family members. The literature seems to suggest that high-grade early-onset prostate cancers have a clinically worse outcome than their elderly-onset counterparts. A feature distinguishing young-age from elderly-onset prostate cancer is the more frequent activation of the androgen-androgen receptor axis, as reflected by the increased frequency of the TMPRSS2-ERG fusion. Some modest progress on the involvement of susceptibility genes has been made in the past few years. This includes the identification of $B R C A 2$-associated prostate cancers with intraductal carcinoma component as an aggressive early-onset category, whereas HOXB13 mutation-associated cancers often have a characteristic histological architecture and a more indolent behaviour. Recognition of unique genetic factors in men with early onset carcinoma and aggressive behaviour can help to target screening and therapy and thus reduce prostate-cancer-specific death among younger men.

\section{Take home messages}

- Young-age prostate cancer has several biological and genetic features, distinct from elderly-onset prostate cancer.

- The majority of patients with prostate cancer at a young age tend to have low-grade and stage disease with superior outcome compared with elderly-onset prostate cancer.

- Early onset prostate cancer has a strong genetic component and represents a subset of young-age and familial prostate cancers with more aggressive disease and higher prostate-cancer-specific death.

- BRCA2 mutation carriers have an increased risk of early-onset prostate cancer with a more aggressive biology, morphologically characterised by the presence of intraductal carcinoma.

- Activation of the androgen-androgen receptor axis manifested by TMPRSS2-ERG fusion mediated over-expression of the $E R G$ protein is a hallmark of young-age low-risk prostate cancer.

\section{Handling editor Runjan Chetty}

Contributors All authors have intellectually contributed to the paper and they all approve of the final submitted version.

\section{Competing interests None.}

Provenance and peer review Commissioned; internally peer reviewed.

\section{REFERENCES}

1 http://www.cancerresearchuk.org/cancer-info/cancerstats/types/prostate/incidence/ \#age

2 Howlader N, Noone AM, Krapcho M, et al., eds. SEER cancer statistics review, 1975-2011. Bethesda, MD: National Cancer Institute, 2014. http://seer.cancer.gov/ csr/1975-2011/

3 Gronberg H. Prostate cancer epidemiology. Lancet 2003;361:859-64.

4 Mitka M. Urology group: Prostate screening should be offered beginning at age 40 . JAMA 2009;301:2538-9.

5 Lilja H, Cronin AM, Dahlin A, et al. Prediction of significant prostate cancer diagnosed 20 to 30 years later with a single measure of prostate-specific antigen at or before age 50. Cancer 2011;117:1210-19. doi:10.1002/cncr.25568 
6 Connolly D, Black A, Gavin A, et al. Baseline prostate-specific antigen level and risk of prostate cancer and prostate-specific mortality: diagnosis is dependent on the intensity of investigation. Cancer Epidemiol Biomarkers Prev 2008;17:271-8.

7 Huben R, Natarajan N, Pontes E, et al. Carcinoma of prostate in men less than fifty years old. Data from American College of Surgeons' National Survey. Urology 1982;20:585-8.

8 Douglas EJ, Lanieri J, Aayala A. Prostatic adenocarcinoma occurring in men under 50 years of age. J Surg Oncol 1972;4:207-16.

9 Lin DW, Porter M, Montgomery B. Treatment and survival outcomes in young men diagnosed with prostate cancer. Cancer 2009;115:2863-71.

10 Wilson JM, Kemp IW, Stein GJ. Cancer of the prostate. Do younger men have a poorer survival rate? Br J Urol 1984;56:391-6.

11 Breslow N, Chan C, Dhom G, et al. Latent carcinoma of prostate at autopsy in seven areas. Int J Cancer 1977;20:680-8.

12 Sakr W, Haas G, Cassin B, et al. The frequency of carcinoma and intraepithelial neoplasia of the prostate in young male patients. J Urol 1993;150:379-85.

13 Stantinos K, Alevizos A, Sofras F, et al. Incidence of impalpable carcinoma of the prostate and of non-malignant and precarcinomatous lesions in Greek male population: An Autopsy study. Prostate 2006;66:1319-28.

14 Sanchez-Chapado M, Olmedilla G, Cabeza M, et al. Prevalence of prostate cancer and prostatic intraepithelial neoplasia in Caucasian Mediterranean males: an autopsy study. Prostate 2003;54:238-47.

15 Soos G, Tsakiris I, Szanto J, et al. The prevalence of prostate carcinoma and it's precursor in Hungary: an autopsy study. Eur Urol 2005;48:739-44.

16 Gronberg $\mathrm{H}$, Damber JE, Jonsson $\mathrm{H}$, et al. Patient age as a prognostic factor in prostate cancer. J Urol 1994;152:892-5.

17 Smith C, Bauer J, Connely R, et al. Prostate cancer in men age 50 years or younger: a review of the department of defense center for prostate disease research multicenter prostate cancer database. J Urol 2000;164:1964-7.

18 Loeb S, Hernandez D, Mangold L, et al. Progression after radical prostatectomy for men in their thirties compared to older men. BJU Int 2008;101:1503-6.

19 Schroder F, Hugosson J, Roobol M, et al. Screening and prostate cancer mortality in a randomized European study. N Eng J Med 2009;360:1320-8.

20 Ali K, Gunnar A, Jan-Erik D, et al. PSA doubling time predicts the outcome after active surveillance in screening-detected prostate cancer: Results from the European randomized study of screening for prostate cancer, Sweden section. Int I Cancer 2006;120:170-4.

21 Kim J, Ebertowski J, Janiga $M$, et al. Many young men with prostate-specific antigen (PSA) screen-detected prostate cancers may be candidates for active surveillance. BJU Int 2013;111:934-40.

22 Ranasinghe W, Kim S, Lawrentschuk N, et al. Population-based analysis of prostate-specific antigen (PSA) screening in younger men ( $<55$ years) in Australia. BJU Int 2014;113:77-83.

23 Powell J, Banerjee M, Sakr W, et al. Should African-American men be tested for prostate carcinoma at an earlier age than white men? Cancer 1999;85:472-7.

24 Powell J, Bock C, Ruterbusch J, et al. Evidence supports a faster growth rate and/o earlier transformation to clinically significant prostate cancer in Black than in White American men and influences racial progression and mortality disparity. J Urol 2010;183:1792-6.

25 Powell J, Vigneau F, Bock C, et al. Reducing prostate cancer racial disparity: evidence for aggressive early prostate cancer PSA testing of African American men. Cancer Epidemiol Biomarkers Prev 2014;23:1505-11.

26 Powell J, Heilbrun LK, Sakr W, et al. The predictive value of race as a clinical prognostic factor among patients with clinically localized prostate cancer: A multivariate analysis of positive surgical margins. J Urol 1997;9:726-31.

27 Heyns CF. Is prostate cancer more common and more aggressive in African men? Afr J Urol 2006;14:66-74
28 Pakkanen S, Baffoe-Bonnie AB, Matikainen MP, et al. Segregation analysis of 1,546 prostate cancer families in Finland shows recessive inheritance. Hum Genet 2007;121:257-67.

29 Zeegers MP, Jellema A, Ostrer $\mathrm{H}$. Empiric risk of prostate carcinoma for relatives of patients with prostate carcinoma: a meta-analysis. Cancer 2003;97: 1894-903.

30 Kiciński M, Vangronsveld J, Nawrot TS. An epidemiological reappraisal of the familial aggregation of prostate cancer: a meta-analysis. PLOS ONE 2011;6:e27130.

31 Gronberg $\mathrm{H}$, Bratt $\mathrm{O}$, Damber JE, et al. Hereditary prostate cancer: clinical characteristics and survival. J Urol 2002;167:2423-6.

32 Lange $E$, Johnson A, Wang $Y$, et al. Genome-wide association scan for variants associated with early-onset prostate cancer. PLOS ONE 2014;9:e93436.

33 Lange E, Salinas C, Zuhlke K, et al. Early onset prostate cancer has a significant genetic component. Prostate 2012;72:147-56.

34 Salinas CA, Tsodikov A, Ishak-Howard $M$, et al. Prostate cancer in young men: an important clinical entity. Nat Rev Urol 2014;6:317-23.

35 Mersch J, Jackson MA, Park $\mathrm{M}$, et al. Cancers associated with BRCA1 and BRCA2 mutations other than breast and ovarian. Cancer 2015;121:269-75.

36 Mitra A, Fisher C, Foster CS, et al. Prostate cancer in male BRCA1 and BRCA2 mutation carriers has a more aggressive phenotype. Br J Cancer 2008;98: 502-7.

37 Edwards S, Kote-Jarai Z, Meitz J, et al. Two percent of men with early-onset prostate cancer harbor germline mutations in the BRCA2 gene. Am J Hum Genet 2003;72:1-12.

38 Kote-Jarai Z, Leongamornlert D, Saunders E, et al. BRCA2 is a moderate penetrance gene contributing to young-onset prostate cancer: implications for genetic testing in prostate cancer patients. Br J Cancer 2011;105:1230-4.

39 Sigurdsson S, Thorlacius S, Tomasson J, et al. BRCA2 mutation in Icelandic prostate cancer patients. J Mol Med 1997;75:758-61.

40 Castro E, Goh C, Olmos D, et al. Germline BRCA mutations are associated with higher risk of nodal involvement, distant metastasis, and poor survival outcomes in prostate cancer. J Clin Oncol 2013;31:1748-57.

41 Risbridger G, Taylor R, Clouston D, et al. Patient derived xenografts reveal that intraductal carcinoma of prostate is a prominent pathology in BRCA2 mutation carriers with prostate cancer and correlates with poor prognosis. Eur Urol 2015;76:496-503.

42 Ewing C, Anna M, Ray MS, et al. Germline mutations in HOXB13 and prostate cancer risk. N Engl J Med 2012;366:141-9.

43 Laitinen V, Wahlfors T, Saaristo L, et al. HOXB13 G84E mutation in Finland: Population-based analysis of prostate, breast, and colorectal cancer risk. Cancer Epidemiol Biomarkers Prev 2013;22:452-60.

44 Karlsson R, Aly M, Clements $M$, et al. A population-based assessment of germline HOXB13 G84E mutation and prostate cancer risk. Eur Urol 2014;65:165-76.

45 Smith S, Palanisamy N, Zuhlke K, et al. HOXB13 G84E-related Familial prostate cancers a clinical, histologic, and molecular survey. Am J Surg Pathol 2014;38:615-26.

46 Weischenfeldt J, Simon R, Feuerbach L, et al. Integrative genomic analyses reveal an androgen-driven somatic alteration landscape in early-onset prostate cancer. Cancer Cell 2013;23:159-70.

47 Schaefer G, Mosquera JM, Ramoner $\mathrm{R}$, et al. Distinct ERG rearrangement prevalence in prostate cancer: higher frequency in young age and in low PSA prostate cancer. Prostate Cancer Prostatic Dis 2013;16:132-8.

48 Huang KC, Dolph M, Donnely $B$, et al. ERG expression is associated with increased risk of biochemical relapse following radical prostatectomy in early onset prostate cancer. Clin Trans/ Oncol 2014;16:973-9.

49 Steurer $\mathrm{S}$, Mayer $\mathrm{P}, \mathrm{Adam} \mathrm{M}$, et al. TMPRSS2-ERG fusions are strongly linked to young patient age in low-grade prostate cancer. Eur Urol 2014;66:978-81. 